\title{
TESTING AND CALIBRATING AN ADVANCED CUBESAT ATTITUDE DETERMINATION AND CONTROL SYSTEM
}

\author{
Tjorven Delabie $^{(1)}$, Dirk Vandepitte ${ }^{(1)}$, Bart Vandenbussche $^{(2)}$ \\ (1) KU Leuven, Department of Mechanical Engineering, Celestijnenlaan 300, 3001 Heverlee, \\ tiorven.delabie@kuleuven.be, dirk.vandepitte@kuleuven.be \\ (2) KU Leuven, Institute of Astronomy, Celestijnenlaan 200D, 3001 Heverlee, \\ bart.vandenbussche@kuleuven.be
}

\begin{abstract}
CubeSats are increasingly used for more complex missions. With rising mission complexity, the requirements on the attitude determination and control system get more stringent. To deliver subdegree pointing accuracy, CubeSat ADCS systems now incorporate reaction wheels and a star tracker. An example is the ADCS developed at the KU Leuven university, that delivers a pointing accuracy of around 0.1 degree to $3 \mathrm{U}-6 \mathrm{U}$ CubeSats. To achieve this performance, the ADCS components need to be properly calibrated. On top of that, the ADCS should be adequately tested to verify that the components can reach the desired lifetime. This paper will discuss a number of tests and calibration steps taken in the development of the KU Leuven ADCS, within the SIMBA CubeSat mission.
\end{abstract}

\section{INTRODUCTION}

CubeSats are used for increasingly complex missions, and the need for more accurate and reliable attitude determination and control grows as a result. This system allows the spacecraft to orient itself in space in order to point its payload, solar panels and antennas in the right direction. The ADCS is a complex and often highly autonomous system that consists of a number of different subsystems that together point the spacecraft. Sensors and actuators on the hardware side and controllers and estimators on the software side need to work together for the ADCS to be able to carry out its tasks. A comprehensive test campaign is imperative to reduce risk.

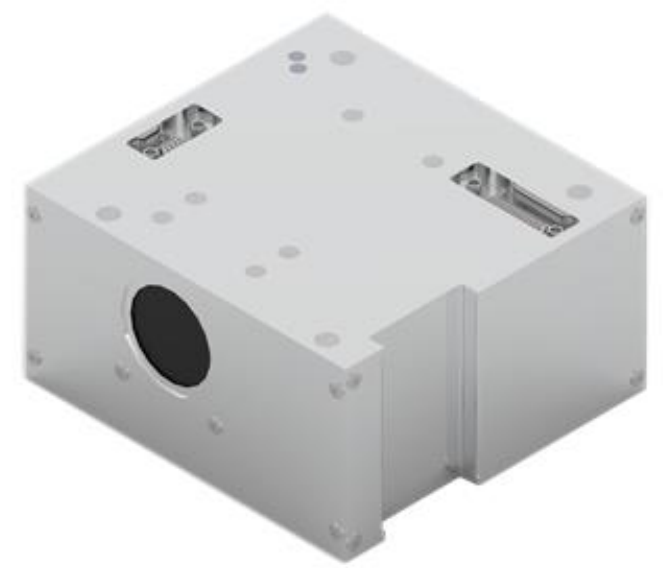

Figure 1 The KU Leuven CubeSat ADCS (50mm high) 
At KU Leuven, we have developed a 0.5U ADCS that fits on the CubeSat stack and gives high accuracy attitude determination and pointing to CubeSats. This ADCS was developed within the ESA-overseen SIMBA mission and has been thoroughly tested, both functionally as environmentally. During these tests, the ECSS rules were followed where possible.

As the pointing requirements of CubeSats get more stringent, calibrating the ADCS becomes increasingly important. The developed ADCS went through a number of calibration steps before launch and also has procedures implemented to calibrate the ADCS on orbit. In this paper, we will focus on the tests and calibration steps of the ADCS subsystems that are most crucial to deliver the envisioned pointing accuracy of around $0.1 \mathrm{deg}$ : The reaction wheels and the star tracker.

The reaction wheels are often the limiting factor in ADCS lifetime. The bearings that support the flywheel can experience increased friction over time and may eventually fail, resulting in a failure of the reaction wheel. We have carried out extensive lifetime tests in thermal vacuum to verify that the reaction wheels have a sufficiently long lifetime for the SIMBA mission and most typical CubeSat missions in general. On top of that, we carried out vibration tests to verify that the reaction wheels survive the launch environment of the typically used CubeSat launchers.

The star tracker is inherently the most accurate sensor of the ADCS and gives the system its high attitude determination accuracy. Given the compact dimensions, we are limited to using small-scale optics, which are more prone to optical distortions. Proper calibration of these distortions can greatly increase the accuracy of the attitude knowledge. The calibration procedure to do this is described in this paper.

The trend towards higher pointing accuracy and agility is expected to hold in the future. With potentially longer and more demanding interplanetary missions, the need for thorough testing and calibration will only increase further. The star tracker will most likely be a crucial component in any interplanetary CubeSat.

\section{REACTION WHEEL LIFETIME TESTING}

Reaction wheels give CubeSats agile attitude control and are necessary in most CubeSat missions. Since these components contain a fast-spinning reaction wheel, supported by bearings, they are prone to wear and eventually failure. Depending on the design, component selection and usage of the wheels, the reaction wheel lifetime can be significantly increased. To verify that the reaction wheels developed at KU Leuven have a large enough lifetime for the SIMBA mission and most other CubeSat missions (> 3 years), the wheels were lifetime tested.

\subsection{Lifetime test profile}

Accelerated lifetime tests in thermal vacuum were used to validate the lifetime of the reaction wheels. During these tests, a predefined reaction wheel speed cycle was repeated. To have a representative cycle, all the phenomena that are present in the expected reaction wheel cycles must be taken into account. From the ADCS analysis, we can derive the following phenomena:

- The rotational speed of the reaction wheels fluctuates around the desired rotational speed (in this case $2000 \mathrm{rpm}$ ) with a low frequency and an amplitude in the order of 100-1000 rpm.

- In the worst case example, there is a peak in rotational speed when the spacecraft exits eclipse and the star tracker is not used. Depending on the drift during eclipse, this peak will be higher or lower in amplitude. The change in rotational velocity can be positive or 
negative. Such a peak in rotational speed can also occur during a large maneuver.

- The reaction wheel speed could switch signs. Reversing the rotational rate is an important factor for wear so this should also be present in the reference cycle.

A reference cycle is proposed that simulates 2 days of reaction wheel activity on orbit. The reaction wheel speed peaks are not clearly present after every eclipse because there are times when the accumulated drift after eclipse is not that high. As a worst case example, we assume a peak with varying amplitude (up to $6000 \mathrm{rpm}$ ) after every two eclipses (14 orbits/day assumed). Furthermore, during the entire sequence, when there are no peaks, the controller assures that the rotational velocity fluctuates in a range around $2000 \mathrm{rpm}$ (no smooth rotational velocity) with a similar amplitude as is analysed. On top of that, we simulate that 1 peak per day leads to a switch of sign of rotational rate. To lead to a continuous cycle, where the end and beginning of the cycle is the same, we take a cycle that covers 2 days (so that there are two switches of sign).

\subsection{Lifetime test duration}

The reference cycle represents 2 days of full usage of reaction wheels on orbit. For a lifetime of 3 years, we would therefore have 548 cycles. This is assuming that the wheels are continuously actuated during the entire lifetime of the mission.

Using the table outlined in ECSS-E-ST-33-01 this leads to a total of 2252 test cycles:

\begin{tabular}{|c|c|c|}
\hline Cycle $1-10$ & $10 \times 10$ & 100 \\
\hline Cycle $11-1000$ & $538 \times 4$ & 2152 \\
\hline
\end{tabular}

Adding 50 cycles on-ground:

\begin{tabular}{|l|c|c|}
\hline Cycle 1-50 & $50 x 4$ & 200 \\
\hline
\end{tabular}

Leads to a total of 2452 cycles.

Simulating 1 cycle takes 970 seconds. The total duration is therefore:

2452 cycles $x 970$ s/cycle $=2.378 .440$ seconds $=27,5$ days

\subsection{Lifetime test setup}

The test setup that was used during the lifetime tests of the reaction wheels is shown in Figure 2. 


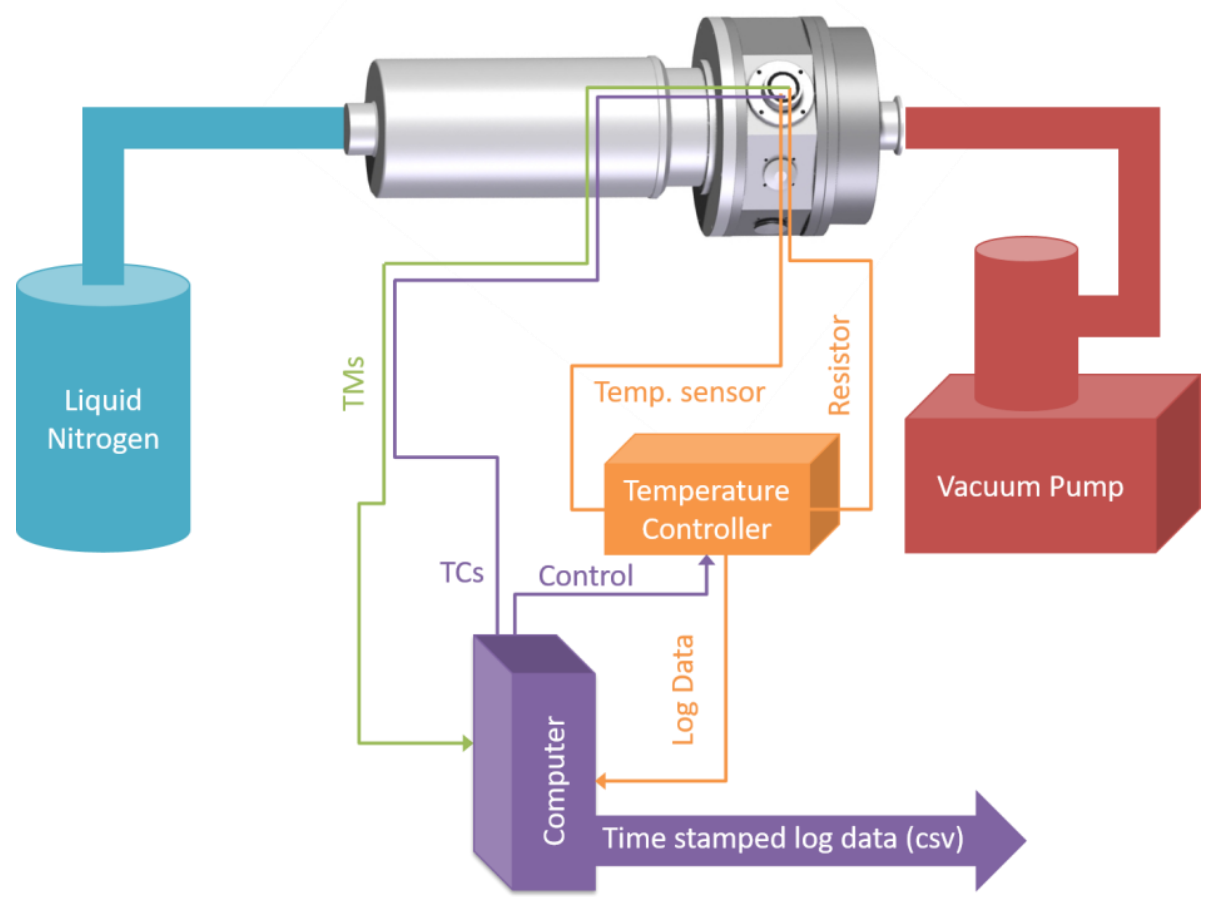

Figure 2 Lifetime Tvac test setup

The reaction wheel was placed in a thermal vacuum chamber. The wheel is actuated to follow the test sequence continuously. An Arduino Uno connected to a pc is used to control and monitor the wheel. The thermal cycles were performed by adding liquid nitrogen to a cooling container. This section is connected to the RW using thermal links. This way, the reaction wheel is cooled down. It is heated up again by a resistor that is placed on the RW adapter plate. Thermal cycles going from -20 deg to +40 deg Celsius were added to the tests.

Since the process of adding liquid nitrogen to the vacuum pump is labour intensive, thermal cycles were not added throughout the entire test time. The temperature profile during a part of these tests is given in the figure below.

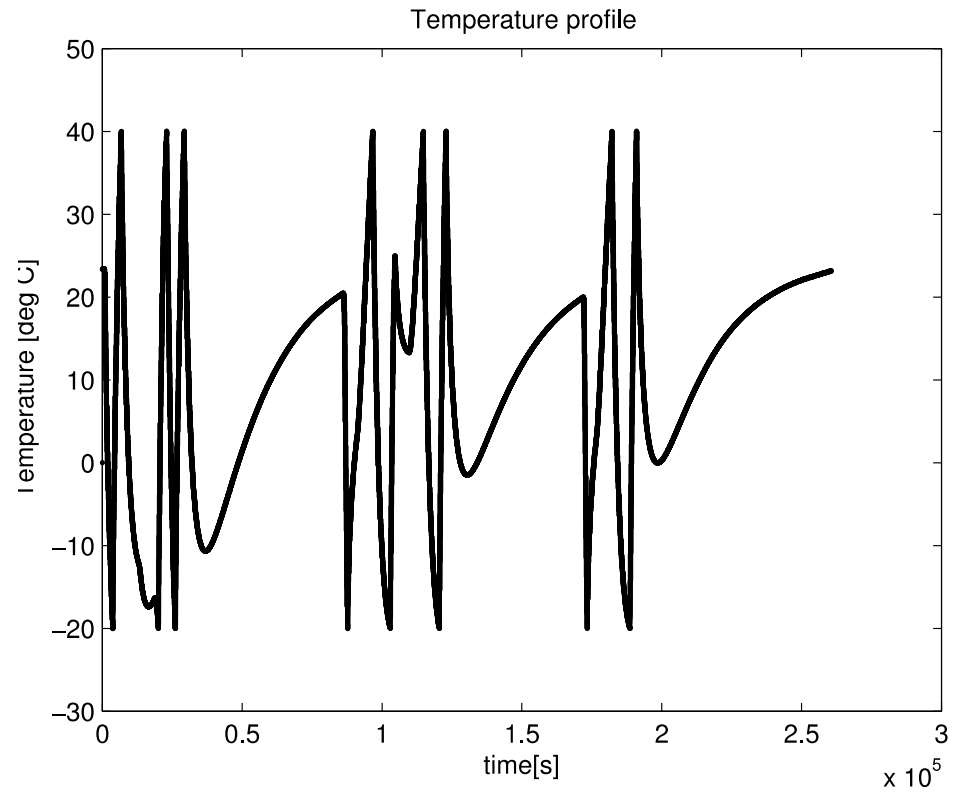

Figure 3 The temperature of the RW during a part of the thermal vacuum tests 


\subsection{Lifetime test results}

A total of 4500 cycles were carried out, largely above the required number of 2452 cycles. The 4500 cycles correspond to a lifetime of over 6 years. An example of one measured cycle can be seen below.

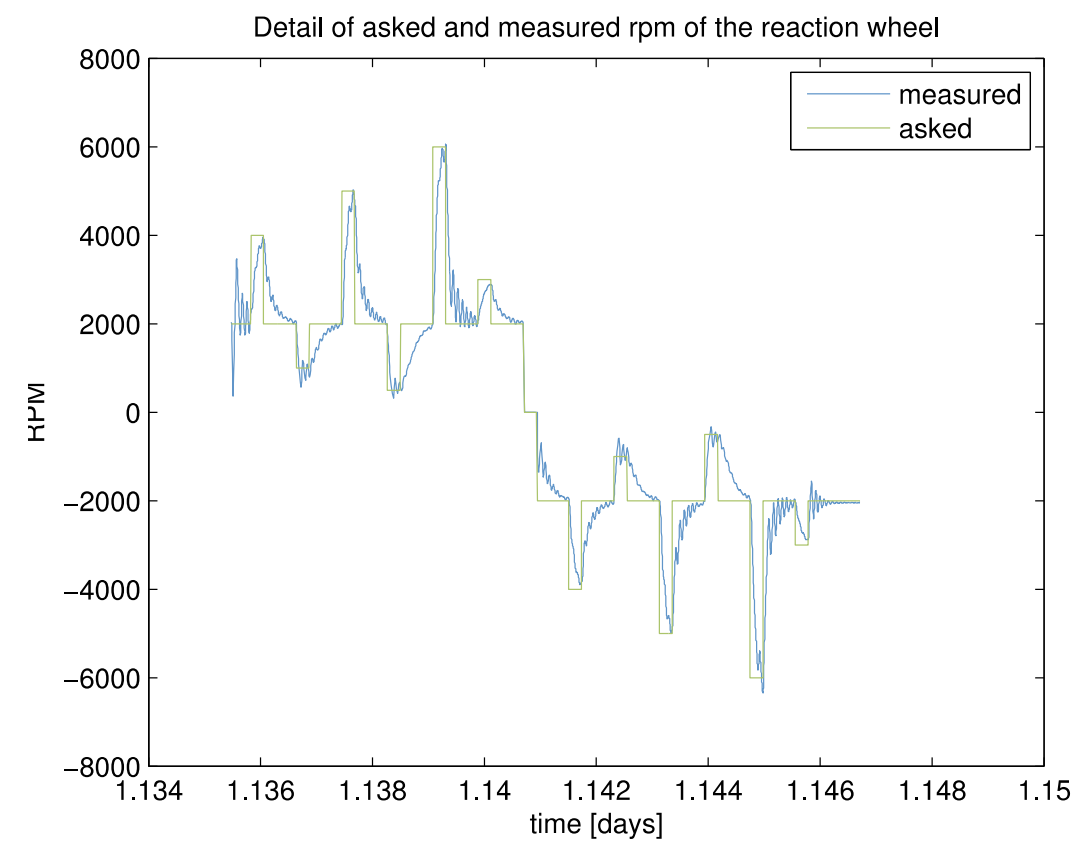

Figure 4 A star tracker lifetime test cycle

The figure below shows different cycles throughout the test sequence plotted on top of each other. Each 50th cycle was plotted. There is no clear degradation and the RW keeps going through the cycle as required.

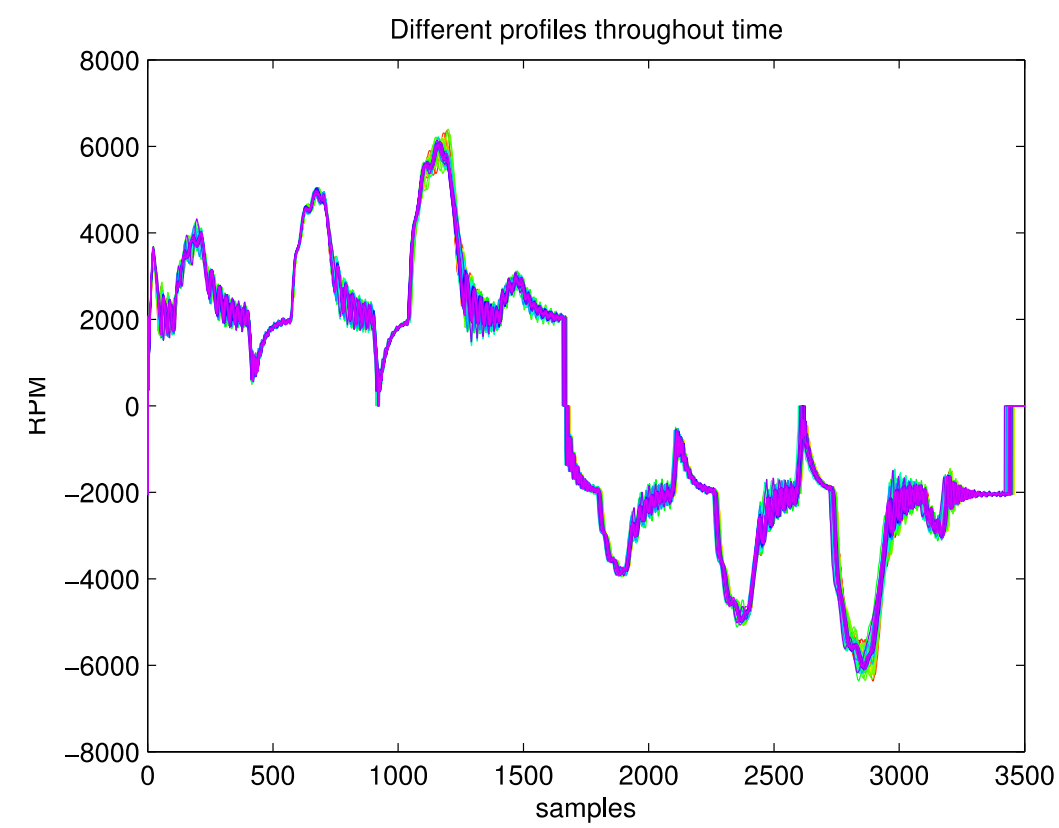

Figure 5 RW cycles plotted on top of each other

At two different times (separated by 3 weeks), a more involved test sequence was used to verify the spindown of the RW. The results of this spindown test are given below. The results are clearly very similar, with the latter test having slightly smaller friction, but the difference is negligible. 


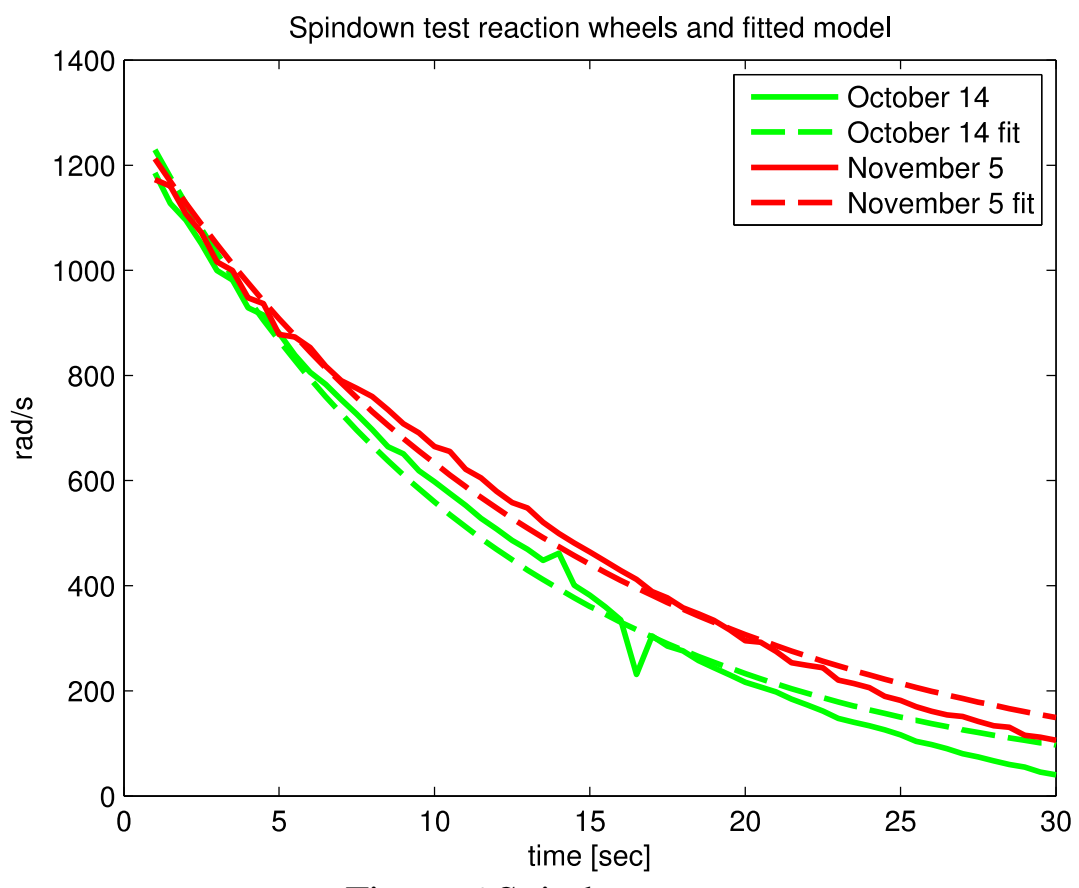

Figure 6 Spindown test

\subsection{Conclusion}

Thermal vacuum tests were carried out with a representative test cycle. Because of the excellent performance of the reaction wheels, the test was carried out longer than envisioned. The reaction wheels were lifetime tested for a period of more than 6 years, after which the test was stopped. The tested reaction wheel showed no sign of deterioration in performance and had no visual signs of wear when it was inspected after the test.

\section{REACTION WHEEL VIBRATION TEST}

The reaction wheel was mounted on a shaker using an adapter plate. The wheel was mounted in three different orientations during different shaking sessions. Before and after the applied vibrations, the wheels were tested.

\subsection{Reaction wheel vibration test procedure}

The reaction wheels are subjected to worst case vibration sequences. The performance and power consumption of the reaction wheel is tested before and after the sequences. It is then checked that performance does not deteriorate to undesirable levels.

The vibration profiles were determined by taking the worst case scenarios for different launchers as analysed in [1]. The reaction wheel performed a certain rotational speed profile before tests, after the sine vibration test and after the random vibration test. It is checked whether the wheels still obtain their maximum angular speed and whether there are significant changes in the profile. The applied vibration spectrum for the sine and random vibrations is given below. 


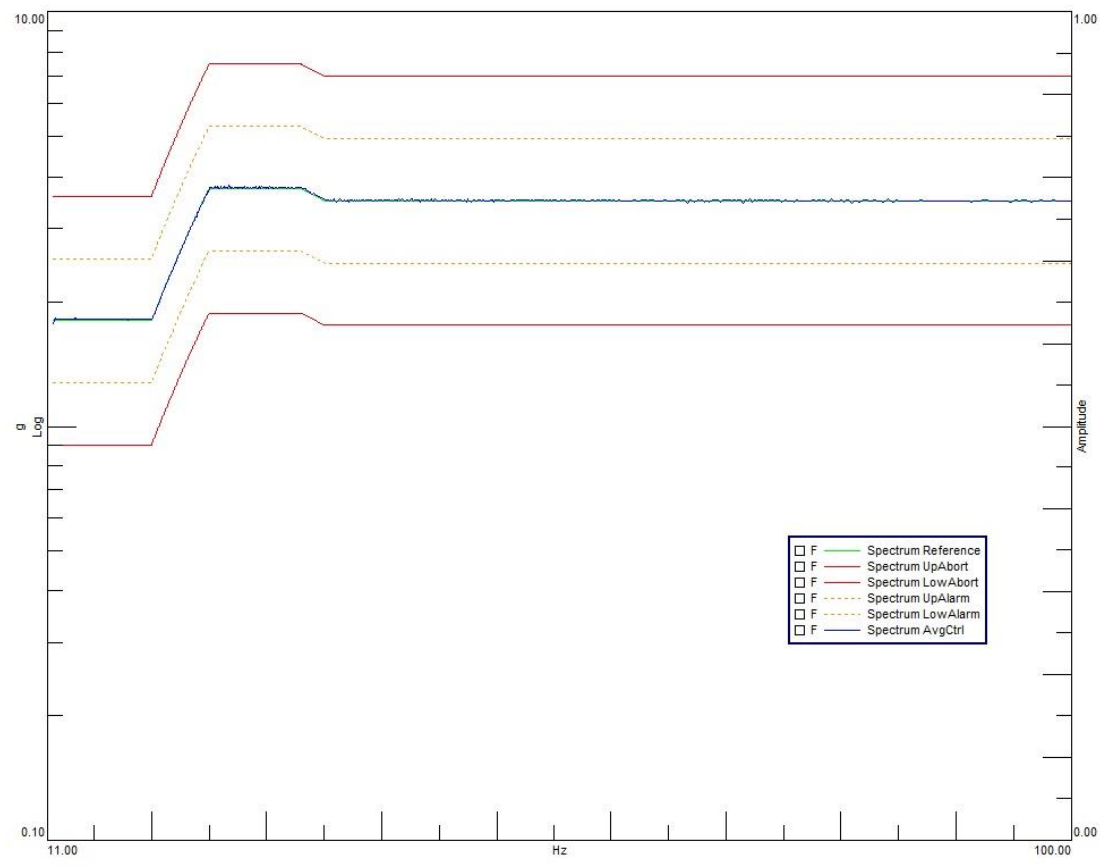

Figure 7 Sine vibration spectrum

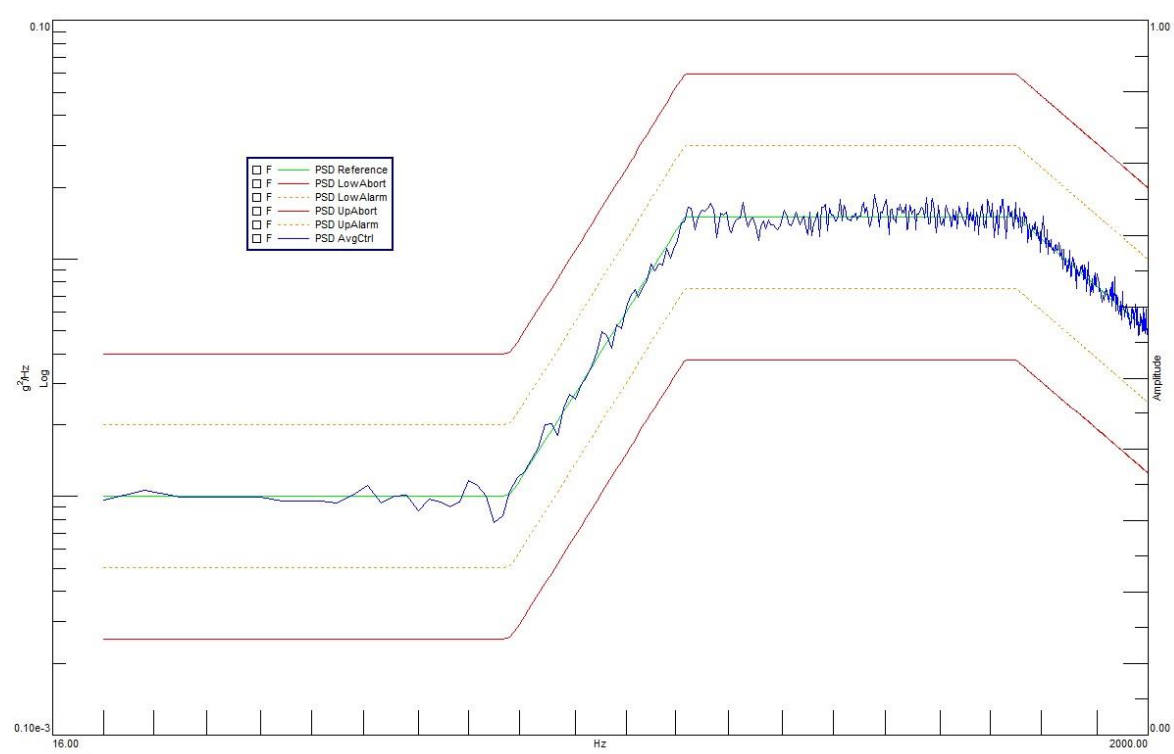

Figure 8 Random vibration spectrum

\subsection{Reaction wheel vibration results}

An example of a reaction wheel speed test before and after vibrations is given below. Analysis shows that there is no significant change in the performance of the reaction wheel after the vibration tests. 


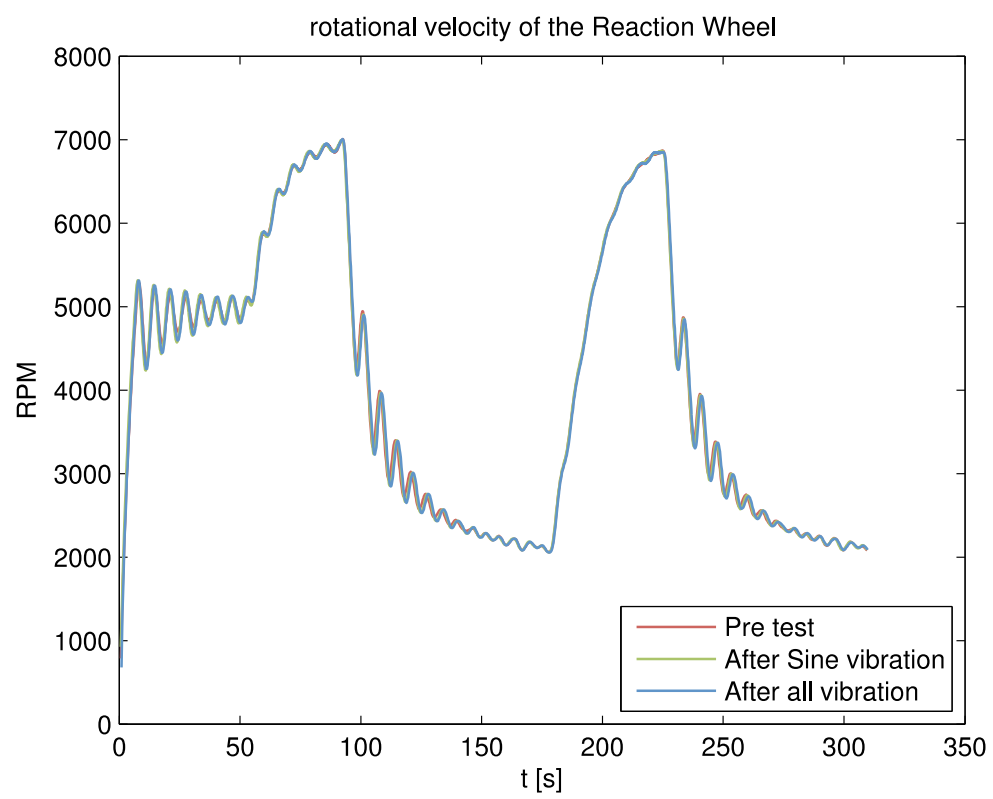

Figure 9 Rotational speed of the reaction wheel before, between and after vibrations

There is no noticeable rise in power consumption. The PWM signal that is given to the reaction wheels remains the same. The rotational speed over PWM signal is for the three sequences given below.

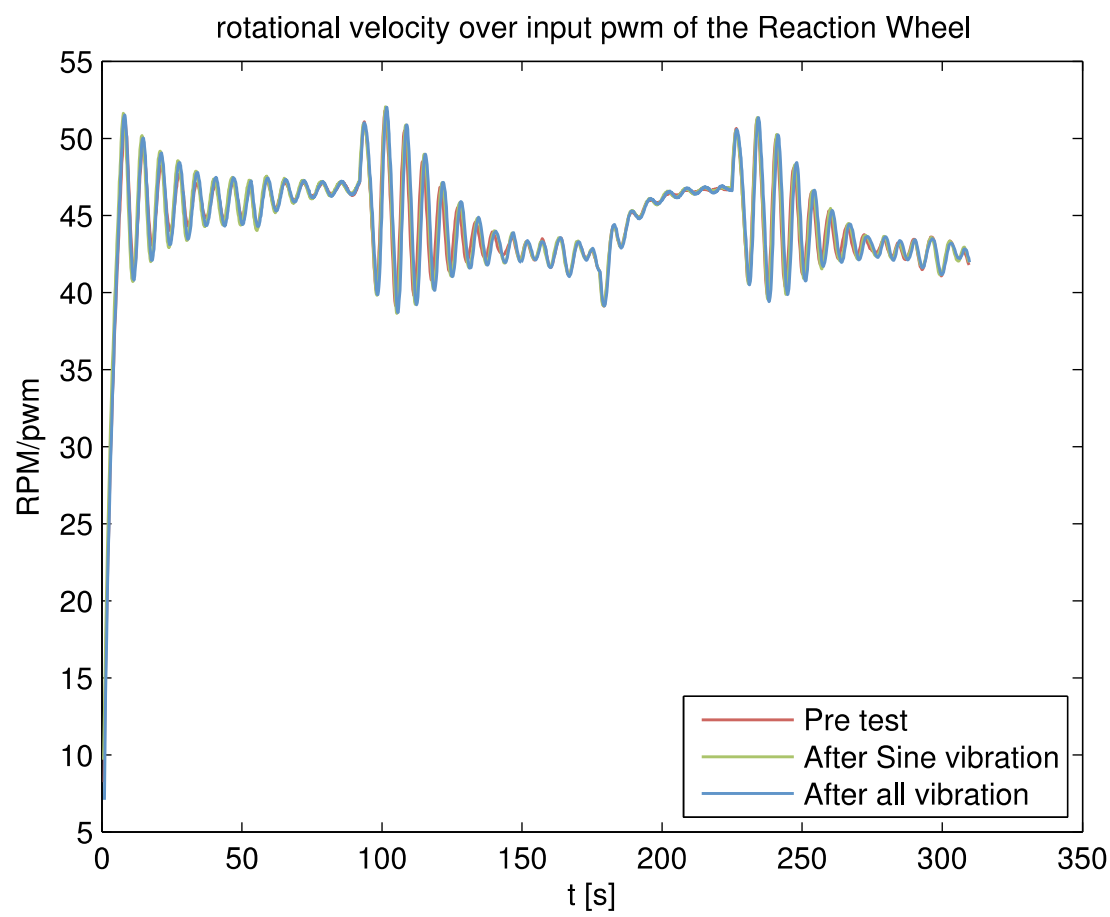

Figure 10 Rotational velocity over pwm signal before, between and after vibrations

\subsection{Conclusion}

The reaction wheels were vibration tested with a combination of worst case loads for typical CubeSat launchers. The wheel was tested before and after the vibration tests and showed no change in performance and functionality due to the vibrations. We can therefore conclude that the reaction wheels successfully withstand the launch environment. 


\section{STAR TRACKER CALIBRATION}

One of the most important error contributors in the star tracker, are the optical distortions. The result of these distortions is that stars are observed at a different location than their true position. Lens distortions are typically more severe, further away from the optical axis. These distortions can be calibrated and correction polynomials can be used to 'correct' the position of the measured star centroid.

\subsection{Calibration procedure}

To be able to correct the optical distortions using correction polynomials, we first need to determine the parameters of these correction polynomials. This is done by measuring the deviation between true and measured star centroids, over the entire image plane. The difficulty here is to have knowledge on the true star positions. In our approach, we use an accurate hexapod and a collimated light beam to generate a star field.

The camera and lens are placed on the hexapod. A collimated light beam is directed at the lens. The hexapod allows the camera and lens to be precisely rotated over a range of 12 deg around the crossboresight axes so that the collimated light beam can go over the full image sensor.

A schematic of the test setup is given below.

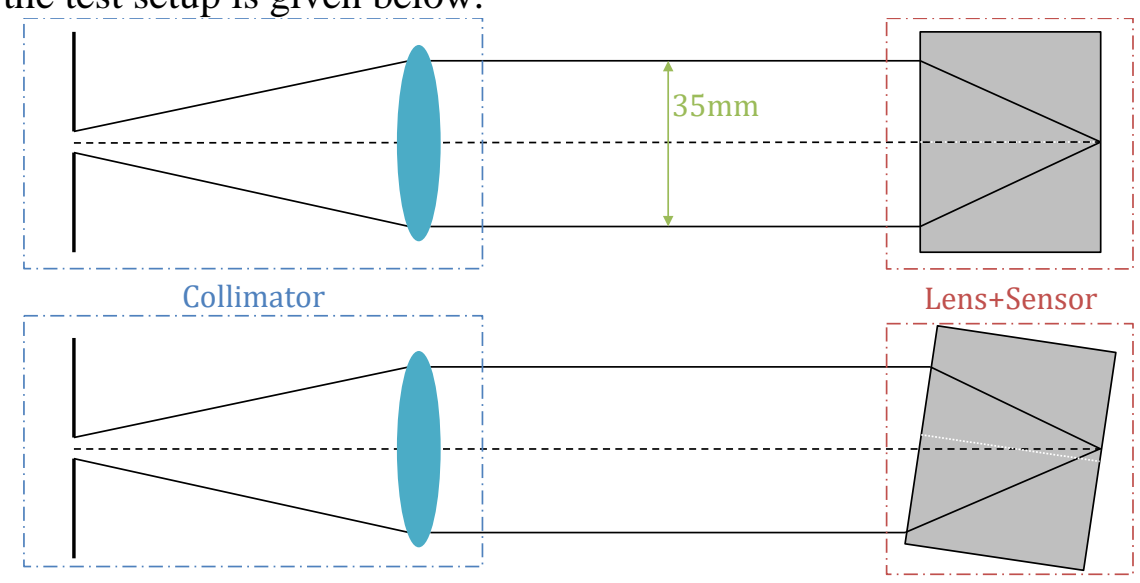

Figure 11 Lens calibration on a hexapod

The camera and lens are mounted on the hexapod. As shown in Figure 12.

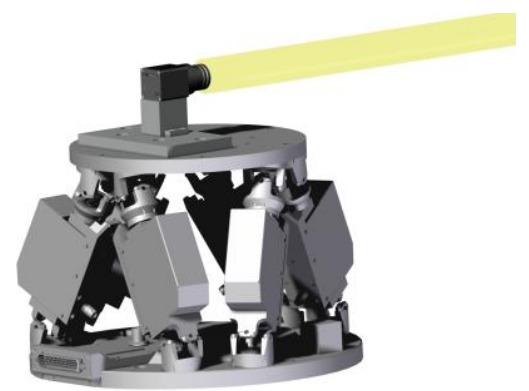

Figure 12 Camera on the hexapod

The hexapod is controlled in such a way that the collimated light beam is directed over the full range of the image plane. An equidistant grid of simulated star measurements is formed in such a way (See Figure 13). For each hexapod orientation, the measured star centroid and the hexapod orientation are stored. 


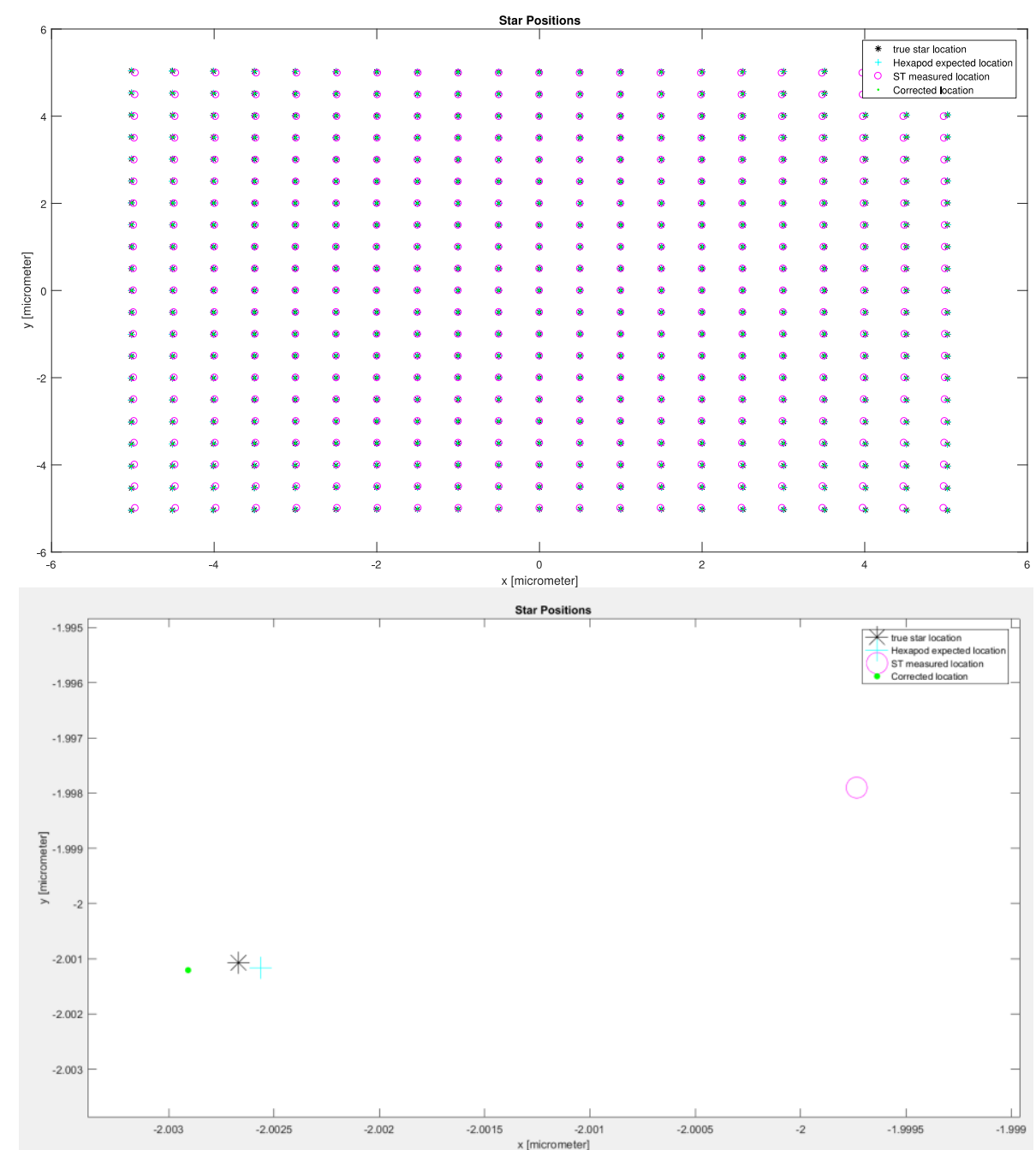

Figure 13 Top: Equidistant grid of star positions (black: True star location, cyan: Hexapod expected location, magenta: Star tracker measured location, green: Corrected location). Bottom: Detail.

The hexapod orientation allows to determine the direction of the star light. In the determination of the polynomials, these values will be considered to give the true orientation of the star light. When compared to the measured star positions, the polynomials can be determined.

The "true" star positions derived from the hexapod orientation and the measured star positions are inserted into an optimization problem. This problem determines the polynomial parameters that optimally maps the measured star positions on the "true" star positions.

\subsection{Calibration simulation setup}

As this test has not yet been carried out on a hexapod, we simulated the test procedure in Matlab.

This simulation creates a grid of star positions using the "true" rotation angles of the hexapod. The star positions created in this way are therefore the positions the stars would fall on, in the case of perfect optics. Next, we add optical distortions to these positions using predetermined optical distortion polynomials. The resulting star positions are the positions where the light will hit the detector in reality, due to lens distortions. 
To obtain the measured star positions, a centroiding error is added to these positions. The resulting star positions are the "ST measured" star positions of the star tracker.

These measured star positions should be compared to the true star positions in order to find the distortion correction polynomial parameters. However, the true star positions are not known in reality. There is noise added since the hexapod does not function perfectly and we therefore do not know the true star location perfectly. To get the "hexapod expected" star positions, hexapod positioning noise and the hexapod calibration error are added to the true angles, which is in turn translated to the "hexapod expected" star positions.

The optimization algorithm is then executed to find the polynomial parameters that optimally map the "ST measured" star positions on to the "hexapod expected" star positions. The obtained polynomials are then used to translate the "ST measured" star positions to the "corrected star positions".

The following values were used in this simulation:

- Equidistant star grid of $23 \times 23$ star positions

- Focal length of $50 \mathrm{~mm}$

- $\quad$ Number of pixels $=2048 \times 2048$

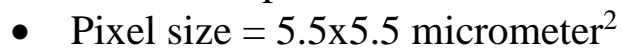

- 1 meter distance between collimated light beam source and image plane

- Centroiding accuracy $=0.05$ pixel

- Hexapod rotation accuracy $=1$ arc seconds

The added optical distortions:

$y=k_{0}+k_{1} y+k_{2} z+k_{3} y r+k_{4} y r^{2}+k_{5} y^{2}+k_{6} y z+k_{7} z^{2}$

$z=h_{0}+h_{1} z+h_{2} y+h_{3} z r+h_{4} z r^{2}+h_{5} z^{2}+h_{6} y z+h_{7} h^{2}$

with

$r=y^{2}+z^{2}$

and

\begin{tabular}{|c|c|c|}
\hline $\begin{array}{c}\text { Polynomial } \\
\text { number }\end{array}$ & $\mathbf{k}$ & $\mathbf{h}$ \\
\hline 0 & $-1.303 \mathrm{e}-4$ & $1.876 \mathrm{e}-5$ \\
\hline 1 & 0.99999929 & 0.9999996 \\
\hline 2 & $-8.512 \mathrm{e}-5$ & $1.617 \mathrm{e}-5$ \\
\hline 3 & $-2.187 \mathrm{e}-4$ & $2.146 \mathrm{e}-4$ \\
\hline 4 & $6.327 \mathrm{e}-7$ & $3.481 \mathrm{e}-7$ \\
\hline 5 & $-6.415 \mathrm{e}-5$ & $-4.463 \mathrm{e}-5$ \\
\hline 6 & $-5.213 \mathrm{e}-5$ & $-4.801 \mathrm{e}-5$ \\
\hline 7 & $-2.204 \mathrm{e}-5$ & $-2.234 \mathrm{e}-5$ \\
\hline
\end{tabular}

These values were taken from the Herschel star tracker calibration [2]. 


\subsection{Calibration simulation results}

For every simulation case, 1000 images are simulated. The RMS error on star positions is given below:

\begin{tabular}{|c|c|c|}
\hline Scenario & Rms error x (pixel) & Rms error y (pixel) \\
\hline ST measured & 3.94 & 4.08 \\
\hline Corrected & 0.063 & 0.092 \\
\hline Corrected known & 0.061 & 0.078 \\
\hline
\end{tabular}

Without distortions, we expect the RMS centroiding error to be 0.05 pixels. The result with lens distortions and without distortion correction (ST measured) determines the star positions with an accuracy of around 4 pixels rms. The distortion parameters were taken from the Herschel star tracker calibration and can be expected to give a good order of magnitude estimate of optical distortions in these types of optical instruments.

After correction, the error is reduced to below 0.1 pixel (Corrected). This is an addition of below 0.05 pixel error on the ideal 0.05 error due to inaccurate centroiding. However, since in reality we will not know the actual "true" positions, we can only assess the improvement compared to the "hexapod expected" positions. Compared to these, we would be led to believe the error is around 0.07 pixels (Corrected known).

With a star position accuracy of 0.1 pixel, we can expect a star tracker accuracy in our case of:

$$
N E A=\frac{E_{\text {cent }} F O V}{N_{\text {pix }} \sqrt{N_{\text {stars }}}}=\frac{0.1 * 12}{2048 * \sqrt{16}}=0,53 \text { arc seconds }
$$

If the optical distortions are larger, we can expect to have a larger remaining error. To test this, we multiplied all distortion parameters (expect $\mathrm{k}_{1}$ and $\mathrm{h}_{1}$ ) by a factor.

\section{Factor 2}

\begin{tabular}{|c|c|c|}
\hline Scenario & Rms error x (pixel) & Rms error y (pixel) \\
\hline ST measured & 7.45 & 7.70 \\
\hline Corrected & 0.12 & 0.10 \\
\hline Corrected known & 0.07 & 0.08 \\
\hline
\end{tabular}

\section{Factor 5}

\begin{tabular}{|c|c|c|}
\hline Scenario & Rms error x (pixel) & Rms error y (pixel) \\
\hline ST measured & 18.6 & 19.2 \\
\hline Corrected & 0.19 & 0.15 \\
\hline Corrected known & 0.08 & 0.08 \\
\hline
\end{tabular}

With distortion parameters that are 5 times higher than that of Herschel, we get a final centroiding error of around 0.20 pixel. This leads to a star tracker accuracy of around 1 arc second, which is the threshold we wish to remain under. To visualize the distortion that leads to this result, we show a star grid and a checkerboard pattern that was deformed with this distortion. For the grid, the magenta stars are the measured stars, the green stars are the correct star positions. It is clear that the distortions further away from the optical axes (at the edge of the image) are severe in this simulation case. 


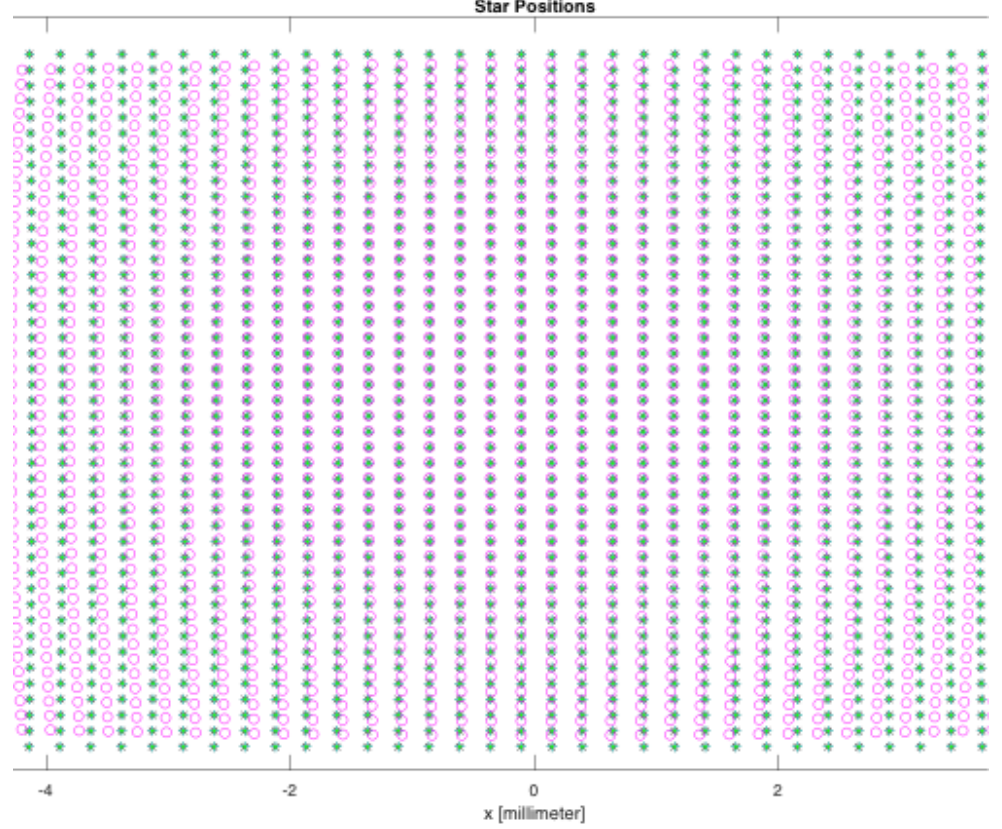

Figure 14 Star grid with higher distortions (factor 5)

Figure 15 gives a visual representation of an image with the distortions of the latter case.

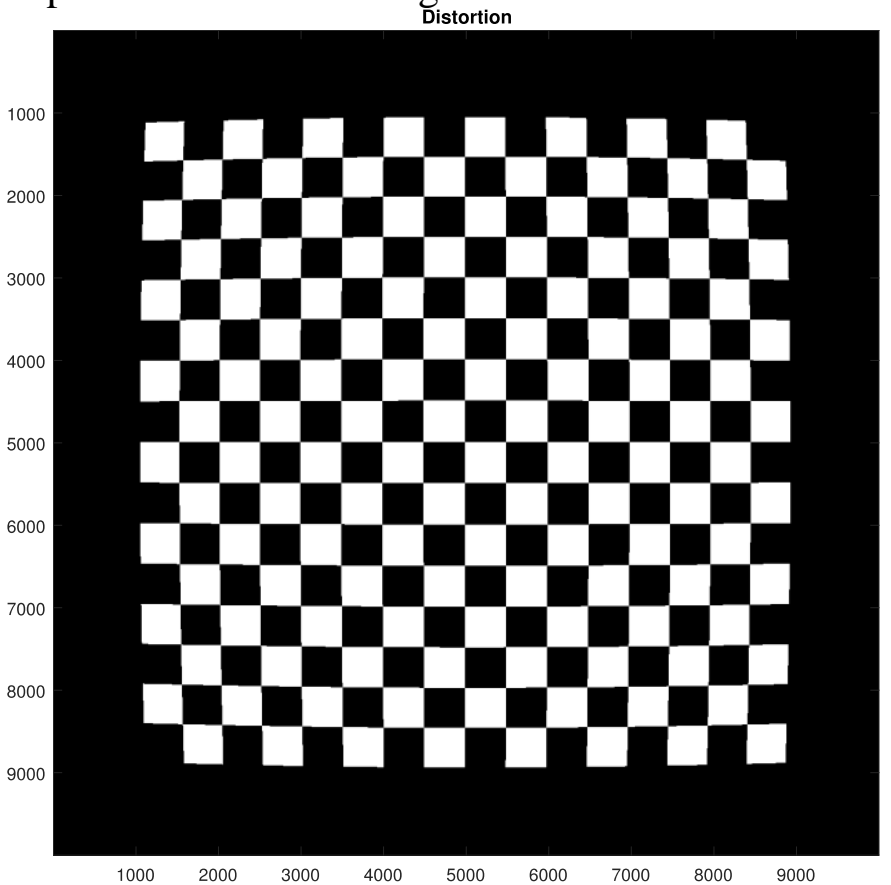

Figure 15 Checkerboard with higher distortions (factor 5)

\subsection{Conclusion}

A calibration procedure to calibrate star tracker lens distortions was developed. The simulation results show that the calibration procedure leads to correction polynomials that significantly improve the centroiding accuracy of the star tracker. The lens distortions are corrected successfully and improve the star tracker accuracy greatly. With realistic values for lens distortions, the calibration procedure manages to keep the star tracker accuracy around 1 arc second. 


\section{CONCLUSION}

This paper discussed some of the tests and calibration steps that were carried out on the KU Leuven ADCS. We focussed on the star tracker and reaction wheels, as these are the components that give the ADCS high pointing precision and agility. The reaction wheels were successfully lifetime tested for a lifetime of around 6 years in thermal vacuum. The vibration tests show that launch vibrations do not significantly impact the reaction wheel performance. The behaviour of the reaction wheels before and after launch is unaltered. The star tracker used in CubeSats is prone to optical distortions. We have discussed our procedure to characterize and compensate these distortions. Simulations show that the centroiding accuracy improves with an order of magnitude thanks to this calibration. The tests presented in this paper, combined with a large number of other functional and environmental tests show that the ADCS can withstand the launch and space environment for the entire SIMBA mission duration. The star tracker calibration procedure will ensure that the required pointing knowledge can be met with large margin.

\section{REFERENCES}

[1] M. Theybers, "Vibration Testing of the Reaction Wheel for the B3LSat," KU Leuven, 2013.

[2] H. Feuchtgruber, "Herschel STR-A CCD Sub-Pixel Structure," 2012. 